\title{
Effect of high dose dexamethasone on platelets apoptosis in children with Chronic Immune Thrombocytopenic Purpura
}

\author{
Asmaa Zahran ${ }^{1}$, Khalid Elsayh², Mona Soliman ${ }^{3}$, and Ismail Mohamad² \\ ${ }^{1}$ South Egypt Cancer Institute, Assiut University, Egypt \\ ${ }^{2}$ Assiut University Faculty of Medicine \\ 3 alazhar university
}

July 2, 2020

\begin{abstract}
Approximately 10 to 20 percent of children who present with ITP develop chronic ITP, defined as platelet count $<100,000 /$ micro L lasting beyond 12 months from the time of presentation. Recently, it was found that apoptosis may be one of the factors controlling the survival and lifespan of the platelets in chronic ITP patients. High dose dexamethasone (HD-DXM) was suggested as one of the effective modalities of in treatment of chronic ITP. In this research we aim to detect the level of apoptotic platelets in children with chronic ITP and to evaluate the effect of high dose dexamethasone on platelets apoptosis in these children. Patients and methods: twenty- six chronic ITP patients and twenty-one age and sex matched healthy volunteers were exposed to quantitative assessment of apoptotic platelets. HD-DXM was given to the patients according to the treatment protocol with reassessment of the apoptotic platelets after the end of the treatment. Results: the mean levels of apoptotic platelets were significantly higher in patients before treatment compared to control and when compared to patients after treatment with HD-DXM. Conclusion: platelet apoptosis may play a role in the pathogenesis of chronic ITP with good impact of HD-DXM on decreasing apoptosis and increasing platelet count.
\end{abstract}

Effect of high dose dexamethasone on platelets apoptosis in children with Chronic Immune Thrombocytopenic Purpura

Asmaa M. Zahran ${ }^{1}$, Khalid I. Elsayh², Mona M Soliman³, Ismail L. Mohamad²

${ }^{1}$ Department of clinical pathology, South Egypt Cancer Institute, Assiut, Egypt.

${ }^{2}$ Pediatric department, faculty of medicine, Assiut University, Assiut, Egypt.

${ }^{3}$ Department of clinical pathology, Faculty of Medicine, Al-Azhar University, Egypt.

* Address correspondence to Ismail Lotfy Mohamad, Associate Professor of pediatrics, Assiut University, Assiut 71516, Egypt. Tel +20-106-339-8967, Fax +20-88-236-8371. E-mail: drsomaa@yahoo.com , ismail231@aun.edu.eg

Abbreviations :

ITP: Immune Thrombocytopenic Purpura, HD-DXM: High dose dexamethasone, SR: sustained response

Abstract

Approximately 10 to 20 percent of children who present with ITP develop chronic ITP, defined as platelet count $<100,000 /$ micro L lasting beyond 12 months from the time of presentation. 
Recently, it was found that apoptosis may be one of the factors controlling the survival and lifespan of the platelets in chronic ITP patients. High dose dexamethasone (HD-DXM) was suggested as one of the effective modalities of in treatment of chronic ITP. In this research we aim to detect the level of apoptotic platelets in children with chronic ITP and to evaluate the effect of high dose dexamethasone on platelets apoptosis in these children.

Patients and methods: twenty- six chronic ITP patients and twenty-one age and sex matched healthy volunteers were exposed to quantitative assessment of apoptotic platelets. HD-DXM was given to the patients according to the treatment protocol with reassessment of the apoptotic platelets after the end of the treatment.

Results: the mean levels of apoptotic platelets were significantly higher in patients before treatment compared to control and when compared to patients after treatment with HD-DXM.

Conclusion: platelet apoptosis may play a role in the pathogenesis of chronic ITP with good impact of HD-DXM on decreasing apoptosis and increasing platelet count.

\section{Introduction}

Immune thrombocytopenia (ITP) is an acquired autoimmune disease in which an abnormal response of humoral and cellular immunity occurs due to defects in self-tolerance that results in autoantibody production and cytotoxic effects.[1]

The defect in immunological pattern leads to platelet destruction, decreases megakaryopoiesis and thrombopoiesis that results in thrombocytopenia. [2], [3]

Approximately 10 to 20 percent of children who present with ITP develop chronic ITP, defined as platelet count $<100,000 /$ micro L lasting beyond 12 months from the time of presentation. Chronic ITP cannot be predicted at diagnosis, but some factors can be associated with increased risk include older age, higher presenting platelet count at the initial diagnosis, insidious onset of symptoms, and lack of preceding infection or vaccination prior to development of ITP [5]

Apoptosis is the process of programmed cell death, which is an important process whereby cells are cleared from the body intentionally marked and is the main mechanism that regulates the life span of the cell and the elimination of damaged or infected nucleated cells. Although platelets are a nucleate, previous studies have shown that they contain the necessary components and the intrinsic program for apoptosis, which controls their survival and dictates their lifespan $[6],[7]$.

In recent years, It was obvious that apoptotic-like sequence occur in platelets and are linked to the production of platelets and their subsequent life span, Moreover studies also suggest that antiplatelet antibodies that induce thrombocytopenia in Murine models are related to increases in apoptotic markers in platelets [ 7].

Winkler et al demonstrated an enhancement of apoptotic processes in the platelets of pediatric patients with acute ITP which was ameliorated by intravenous administration of immunoglobulin. These findings suggest that apoptosis might also contribute to the pathogenesis of ITP [8].

In children with chronic ITP that is not responding to initial therapy, high-dose dexamethasone is recommended as appropriate second-line therapy for those with significant bleeding despite initial therapy, as an alternative to splenectomy, or as therapy in patients who did not respond favorably to splenectomy. Oral doses of dexamethasone reviewed in these guidelines include 6 cycles of the drug with a cycle defined as 0.6 $\mathrm{mg} / \mathrm{kg} /$ day for 4 days every 4 weeks. [9]

Andersen in 1994 reported excellent results with limited side effects of high dose dexamethasone in 10 cases of chronic ITP who were refractory to 2 to 6 previous treatments including splenectomy in 6 cases [10]. Cheng et al introduced a single course of HD-DXM for treatment-naive ITP patients and achieved $85 \%$ initial response and $42 \%$ sustained response (SR) at 6 months' follow-up. [11]

2. Patients and methods 


\subsection{Study Design}

This single-center prospective case-control study was carried out in the Pediatric Hematology Unit, Children's Hospital, Assiut University. Twenty-six patients with chronic immune thrombocytopenic purpura were recruited in this study. In addition to Twenty -one healthy children of comparable age and sex were taken as controls. The study was approved by the institutional review board and informed consents were obtained from parents.

All patients and controls were subjected to through history and physical examination with stress upon disease duration, drug intake, bleeding manifestations, organomegaly and lymphadenopathy. In addition to complete blood picture was done by Celltac E automated hematology analyzer, Tokyo, Japan), and detection of platelet apoptosis. The patients were evaluated twice, before starting of HD-DXM and after the end of treatment course of HD-DXM.

\subsection{Inclusion criteria}

1-Age more than 3 years and less than 18 years of both genders.

2-Confirmed diagnosis as chronic ITP: the duration of ITP is more than 12 months according to American Society of Hematology 2019 guidelines for Immune thrombocytopenia.[12]

3- No treatment with corticosteroids or immunomodulating drugs in the last 2 weeks before the study.

\subsection{Exclusion criteria:}

1. Clinical features not compatible with diagnosis of chronic ITP.

2. Severe or life-threatening bleeding at presentation.

\subsection{Flow Cytometric Detection of Platelets Apoptosis.}

Fluorescein isothiocyanate (FITC)-conjugated Annexin V apoptosis detection kit (Biolegend, GmbH, Germany) and, peridinin-chlorophyll-protein (Per-CP)-conjugated CD41 (Becton Dickinson (BD) Biosciences, San Jose, CA, USA) were used for detection of platelet apoptosis. Platelet rich plasma (PRP) were separated from the citrated blood samples by centrifugation at $1000 \mathrm{rpm}$ for 10 minutes at $25^{\circ} \mathrm{C}$. The cells were washed twice with cold BioLegend's Cell Staining Buffer, and then resuspended in Annexin V Binding Buffer at a concentration of $0.5 \times 10^{7}$ cells $/ \mathrm{ml}$. One hundred $\mu \mathrm{l}$ of cell suspension were stained with $10 \mu \mathrm{l}$ of CD41, annexin $\mathrm{V}$ and propidium Iodide. After incubation for $15 \mathrm{~min}$ at room temperature, four hundred $\mu$ of Annexin V Binding Buffer were added. Anti-human IgG isotype-matched negative control was run with each sample. Flow cytometric analysis was done by FACSCalibur flow cytometry with Cell Quest software (BD Biosciences, USA) (Figure1). 

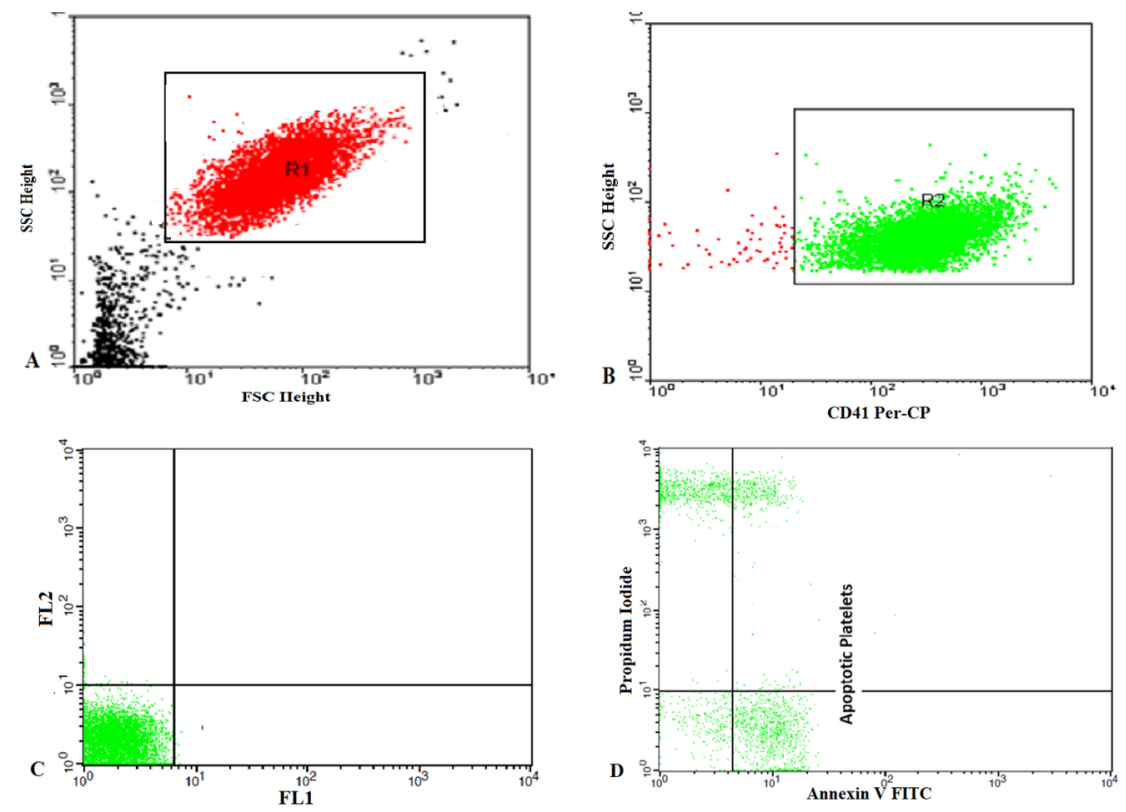

Figure 1: Flow Cytometric Detection of Platelets Apoptosis.

A: Forward and side scatter histogram was used to define the platelets (R1).

B: The expression of CD41 were assessed on R1 to confirm platelets gating (R2).

C: Isotype controls

D: The expression of annexin $\mathrm{V}$ and propidum iodide on $\mathrm{CD} 41^{+}$platelets was assessed to detect apoptotic platelets

\subsection{HD-DXM treatment protocol}

Eligible patients received intravenous dexamethasone $(20 \mathrm{mg} / \mathrm{m} 2)$ daily for 4 days every 15 days for 4 cycles [13]. During the course of treatment, the patients were followed up to avoid any complications during the treatment as measuring the vital signs (pulse, blood pressure), blood glucose and electrolytes, any skin changes or psychic disturbances.

The outcome was measured at the end of the course of treatment by evaluation of apoptotic platelets and platelets count. The definition of complete remission or complete response to treatment is a platelet count [?] $100 \times 109 / \mathrm{L}$ with no clinically relevant bleeding [14].

3. Results

\subsection{Characteristics of the study participants}

The clinical and laboratory findings of the studied cases are shown in table (1).

The mean $\pm \mathrm{SD}$ of the age was $5.65 \pm 2.98$ years, with males $(58 \%)$ were slightly more affected than females (42\%). In the clinical examination of our cases we detected petechial rash and bruising in all cases, epistaxis in 11 cases. No organomegaly detected in any cases. The duration of thrombocytopenia of the cases was 1.2 to 3.5 years.

As regard the laboratory investigations, the mean and SD of platelets was significantly lower in cases (12.09土 4.68) compared to controls (278.39 \pm 76.54$)$, while the mean platelet volume (MPV) was significantly higher 
in cases ( $11.13 \pm 1.99)$ compared to controls $(8.43 \pm 1.57)$. The mean and SD of hemoglobin concentration was significantly lower in cases (10.15 \pm 1.15$)$ compared to controls (11.47 \pm 1.16$)$.

3.2. Table (2) shows that the mean and SD of apoptotic platelets in patients (37.41 \pm 15.98$)$ with chronic ITP was significantly higher compared to controls (7.52 \pm 2.69$)$.

3.3. Table (3) shows the effect of HD-DXM treatment on the apoptotic platelets in chronic ITP patients with significantly lower levels of apoptotic platelets in cases $(9.53 \pm 1.05)$ after treatment compared to before treatment (37.41 \pm 15.98$)$, also the mean \pm SD of platelet count was significantly higher in children with chronic ITP after treatment compered to before treatment.

TABLE 1: Baseline characteristics of chronic ITP patients and controls.

\begin{tabular}{|c|c|c|c|}
\hline $\mathrm{P}$-value & Controls $(n=21)$ & Patients $(n=26)$ & \\
\hline \multirow[t]{8}{*}{0.64} & $6.07 \pm 3.12$ & $5.65 \pm 2.98$ & Age \\
\hline & $13 / 21(62) 8 / 21(38)$ & $15 / 26(58) 11 / 26(42)$ & $\begin{array}{l}\text { Sex Male \% Female } \\
\%\end{array}$ \\
\hline & $0 / 21$ & $26 / 26$ & Petechial rash \\
\hline & $0 / 21$ & $26 / 26$ & Bruising \\
\hline & $0 / 21$ & $11 / 26$ & Epistaxis (mild) \\
\hline & $0 / 21$ & $0 / 26$ & Splenomegaly \\
\hline & $0 / 21$ & $0 / 26$ & Hepatomegaly \\
\hline & & $1.2-3.5$ & $\begin{array}{l}\text { Duration of } \\
\text { thrombocytopenia } \\
\text { (range in years) }\end{array}$ \\
\hline $0.000^{*}$ & $278.39 \pm 76.54$ & $12.09 \pm 4.68$ & $\begin{array}{l}\text { Platelet count } \\
\left(10^{9} / \mathrm{L}\right)\end{array}$ \\
\hline $0.000^{*}$ & $8.43 \pm 1.57$ & $11.13 \pm 1.99$ & $\operatorname{MPV}(\mathrm{fl})$ \\
\hline $0.000^{*}$ & $11.47 \pm 1.16$ & $10.15 \pm 1.15$ & Hemoglobin gm/dl \\
\hline 0.313 & $7.44 \pm 1.96$ & $6.88 \pm 1.85$ & $\begin{array}{l}\text { WBCs count } \\
\left(10^{9} / \mathrm{L}\right)\end{array}$ \\
\hline
\end{tabular}

Data are represented as mean $\pm \mathrm{SD}$, Independent Sample $\mathrm{T}$ test was used in comparison, P[?]0.05 is considered statistically significant

Legend : n, number; L, liter; MPV, mean platelet volume; fl, femtoliter, gram; dl, deciliter; WBCs, white blood cells.

TABLE 2: apoptotic platelet in chronic ITP patients and controls

\begin{tabular}{llll}
\hline Percentage $(\mathbf{\%})$ & Patients $(\mathbf{n}=\mathbf{2 6})$ & Control $(\mathbf{n}=\mathbf{2 1})$ & P-value \\
\hline Apoptotic plt & $37.41 \pm 15.98$ & $7.52 \pm 2.69$ & $0.000^{*}$ \\
\hline
\end{tabular}

Data are represented as mean $\pm \mathrm{SD}$, Independent sample $\mathrm{T}$ test was used in comparison, P[?]0.05 is considered statistically significan $\mathrm{t}$

Legend : n, number, platelet ;reg, regulatory;

TABLE 3: apoptotic platelet in chronic ITP patients pre and post treatment with HD-DXM 


\begin{tabular}{|c|c|c|}
\hline Percentage (\%) & Pre ttt $(n=26)$ & Post ttt \\
\hline 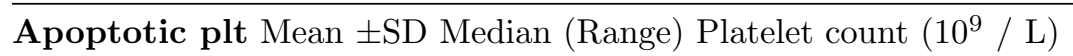 & $37.41 \pm 15.9836 .68(10.6-65) 12.09 \pm 4.68$ & $9.53 \pm 1.0$ \\
\hline
\end{tabular}

Data are represented as mean $\pm \mathrm{SD}$, median(range)Paired $\mathrm{T}$ test was used in comparison, P[?]0.05 is considered statistically significant

Legend : n, number; ttt, treatment; plt, platelet

Discussion

Emerging evidence over the past several years suggests that platelet biogenesis and ageing in chronic ITP are regulated, to some extent, by apoptotic mechanisms. However, the association between decreased platelets and apoptosis in chronic ITP patients is poorly understood. [15]

We tried at this study to clarify the relation between apoptosis and chronic ITP and the effect of HD-DXM on the apoptotic platelets.

Our data detected that the number of platelet apoptosis was increased in patients with chronic ITP when compared to the control group. So, we assume that the observed apoptotic events might play a role in the pathogenesis of thrombocytopenia in chronic ITP. The results obtained in our study were similar to those from previous research by Piguet and Vesin demonstrated that platelet apoptosis may be involved in the development of ITP, which can be induced by pro-apoptotic agonistic antibodies and prevented by apoptosis inhibitors and anti-apoptotic antibodies [16]

In contrast to our findings, Jiaan-Der et al. reported that the percentage of platelets with positive apoptosis markers was not increased in pediatric patients with chronic ITP when compared to controls. This may be due to platelet apoptosis resistance. [17]

HD-DXM was used in our study as one of modalities of treatment of chronic ITP in an attempt to prove its role in increasing the number of platelets by decreasing the process of apoptosis.

We found significant lower levels of apoptotic platelets and higher levels of platelets count in patients with chronic ITP after complete course of treatment with HD-DXM in comparison to patients before treatment.

The same results were recorded by cooper and Bussel in a study done on 2006, that out of 21 patients treated with HD-DXM, 18 patients responded effectively according to the standard definition [18]. Also, Wei et al reported that a high-dose dexamethasone regimen resulted in a higher overall initial response, complete response rates, and shorter time to response with at least comparable long-term prognosis and better tolerance when compared with conventional prednisolone (PDN). HD-DXM also enables patients to avoid the burden of long-term corticosteroids. Therefore, HD-DXM could become a preferred corticosteroid approach for first-line management of adult primary ITP. [19].

The short-term course of HD-DXM in treatment of ITP in addition to decreasing the bleeding manifestations in the patients by lowering the average bleeding score and the number of patients presented by bleeding symptoms, these findings suggest that HD-DXM may be the first choice in treatment of ITP [19].

The mechanism by which HD-DXM can affect the level of apoptotic platelets in chronic ITP is not clear but the changes in the plasma levels of IL-16 and mRNA expression of pro-IL-16, caspase- 3 and T-bet in peripheral blood mononuclear cells (PBMCs) after HD-DXM may provide a new insights into the mechanism for treatment of chronic ITP with HD-DXM [20].

\section{Conclusion}

Platelet apoptosis may play a role in the pathogenesis of chronic ITP with good impact of HD-DXM on decreasing apoptosis and increasing platelet count. 
Acknowledgment : all authors contributed in acquisition, analysis, interpretation of data, all authors designed the study, collected data, analyzed data, created tables and figures, and wrote and revised the article.

\section{Conflict of Interest Statement}

The authors declare that the research was conducted in the absence of any financial relationships that could be construed as a potential conflict of interest.

References

1. McKenzie CG, Guo L, Freedman J, Semple JW. Cellular immune dysfunction in immune thrombocytopenia (ITP). Br J Haematol. 2013; 163:10-23.

2. McMillan R, Wang L, Tomer A, Nichol J, Pistillo J. Suppression of in vitro megakaryocyte production by antiplatelet autoantibodies from adult patients with chronic ITP. Blood. 2004; 103:1364-1369.

3. Lev PR, Grodzielski M, Goette NP, Glembotsky AC, Espasandin YR, Pierdominici MS, et al. Impaired proplatelet formation in immune thrombocytopenia: a novel mechanism contributing to decreased platelet count. Br J Haematol. 2014; 165:854-864.

4. Rosthoj S, Rajantie J, Treutiger I, et al. Duration and morbidity of chronic immune thrombocytopenic purpura in children: five-year follow-up of a Nordic cohort. Acta Paediatr 2012; 101:761.

5. Leytin V. Apoptosis in the anucleate platelet. Blood Rev. 2012;26(2):51-63.

6. Mason KD, Carpinelli MR, Fletcher JI, et al. Programmed a nuclear cell death delimits platelet life span. Cell. 2007;128(6):1173-1186.

7. Leytin V, Mykhaylov S Starkey AF et al Intravenous immunoglobulin inhibits anti-glycoprotein IIbinduced platelet apoptosis in a murine model of immune thrombocytopenia Br J Haematol 2006 , 133(1) , 78-82

8.Winkler J Kroiss S Rand L et al Platelet Apoptosis in pediatric immune thrombocytopenia is ameliorated by intravenous immunoglobulin Br J Hematol 2012 156(4) 508-515.

9.The American Society of Hematology 2011 evidence-based practice guideline for immune thrombocytopenia. Neunert C, Lim W, Crowther M, Cohen A, Solberg L Jr, Crowther MA, American Society of Hematology.Blood. 2011 Apr 21; 117(16):4190-207.

10.Andersen JC. Response of resistant idiopathic thrombocytopenic purpura to pulsed high-dose dexamethasone therapy. N Engl J Med.1994;330(22):1560-1564.

11.Cheng Y, Wong RS, Soo YO. Initial treatment of immune thrombocytopenic purpura with high-dose dexamethasone. N Engl J Med. 2003;349(9):831-836.

12. Cindy Neunert, Deirdra R. Terrell, Donald M. Arnold, George Buchanan, Douglas B. Cines, Nichola Cooper, Adam Cuker, Jenny M. Despotovic, James N. George, Rachael F. Grace, Thomas Kuhne, David J. Kuter, Wendy Lim, Keith R. McCrae, Barbara Pruitt, Hayley Shimanek, Sara K. Vesely. American Society of Hematology 2019 guidelines for immune thrombocytopenia. Blood Adv (2019) 3 (23): 3829-3866.

13. Yadav D, Chandra J, Sharma S, Singh V. Short-course high-dose dexamethasone therapy for chronic idiopathic thrombocytopenic purpura in children. J Trop Pediatr. 2010 Dec; 56(6):446-7.

14 Grace RF, Long M, Kalish LA, Neufeld EJ. Applicability of 2009 international consensus terminology and criteria for immune thrombocytopenia to a clinical pediatric population.

Pediatr Blood Cancer. 2012 Feb; 58(2):216-20.

15.Gang Deng, Shifang Yu, Qiming Li, Yunlei He, Wei Liang, Lu Yu, Deyi Xu, Tao Sun, Ri Zhang, Qiang Li. Investigation of Platelet Apoptosis in Adult Patients with Chronic Immune Thrombocytopenia. Hematology. 2017 Apr;22(3):155-161. 
16. Piguet PF, Vesin C. Modulation of platelet caspases and lifespan by anti-platelet antibodies in mice. Eur J Haematol. 2002;68(5):253-261.

17. Wang JD, Ou TT, Wang CJ, et al. Platelet apoptosis resistance and increased CXCR4 expression in pediatric patients with chronic immune thrombocytopenic purpura. Thromb Res. 2010;126(4):311-318.

18. Cooper N, Bussel J. The pathogenesis of immune thrombocytopaenic purpura. Br J Haematol. (2006) 133:364-74.

19. Wei Y, Ji XB, Wang YW, Wang JX, Yang EQ, Wang ZC, et al. High-dose dexamethasone vs prednisone for treatment of adult immune thrombocytopenia: a prospective multicenter randomized trial. Blood. 2016 Jan 21. 127 (3):296-302; quiz 37

20.Xinru Wang, Lizhen Li, Yuanjian Wang, Xin Li, Qi Feng, Yu Hou, Chunhong Ma, Chengjiang Gao, Ming Hou and Jun Peng. High-Dose Dexamethasone Alters the Increase in Interleukin-16 Level in Adult Immune Thrombocytopenia. Immunol., 18 March 2019.

\section{Hosted file}

Table 1.docx available at https://authorea.com/users/338227/articles/464286-effectof-high-dose-dexamethasone-on-platelets-apoptosis-in-children-with-chronic-immunethrombocytopenic-purpura

\section{Hosted file}

Table 2.docx available at https://authorea.com/users/338227/articles/464286-effectof-high-dose-dexamethasone-on-platelets-apoptosis-in-children-with-chronic-immunethrombocytopenic-purpura

\section{Hosted file}

Table 3.docx available at https://authorea.com/users/338227/articles/464286-effectof-high-dose-dexamethasone-on-platelets-apoptosis-in-children-with-chronic-immunethrombocytopenic-purpura 